\title{
Doctor Franklin's
}

Medicine

Jean-Gaël Barbara

Les États-Unis s'apprêtent à fêter solennellement le tricentenaire de la naissance d'un citoyen exemplaire de Philadelphie, Benjamin Franklin (1706-1790), imprimeur heureux et homme politique, philosophe et médecin à ses heures, mais surtout figure illustre de «l'électricité ». C'est à la suite d'une démonstration publique que Franklin se passionne pour ce sujet, au point d'abandonner l'administration de ses imprimeries pour conduire des expériences qui aboutiront à l'invention du paratonnerre. II parviendra par la suite à marier subtilement une carrière politique internationale, la renommée d'un physicien et celle d'un citoyen réputé dans les affaires médicales et sanitaires.

Stanley Finger $^{1}$ nous livre, par cet ouvrage réussi [1], l'agréable parcours d'une vie de Franklin, révélant son intérêt central pour les aspects médicaux chers à son temps, l'enseignement médical, la création d'hôpitaux pour les pauvres et les aliénés ou, encore, l'adoption de prophylaxies. Franklin n'enrichira pas le domaine médical aussi durablement que celui de la physique. Dans la sphère médicale, son mérite est celui d'un homme attentif au progrès médical, cultivant en Amérique tout savoir dans l'ordre d'une philosophie naturelle pragmatique, avec ce souci de bien faire et de soigner et les autres et lui-même. Cette démarche de Franklin-médecin est en parfait accord avec celle qui prévaut au Siècle des Lumières.

Dans un premier chapitre, Finger rappelle l'intérêt précoce de Franklin pour la médecine, qui fut d'ordre éditorial, avec la publication de son célèbre almanach, un ouvrage transatlantique à succès tiré à 10000 exemplaires annuels, prodiguant aphorismes et conseils médicaux, et ne négligeant pas l'astrologie pour raisons économiques. Ces textes écrits sous un pseudonyme feront sa fortune, et fourniront l'assise financière de sa vie scientifique et politique ultérieure.

Les deux chapitres suivants rassemblent les matériaux des premiers débats médicaux auxquels Franklin participe, la revendication des bienfaits de l'exercice physique, de la nage en particulier, et la polémique concernant les tentatives d'immunisation par inoculation contre la petite vérole. Dans ces deux cas, l'attitude de Franklin est l'adoption immédiate du nouvel esprit de son temps, au point de devenir champion dans l'art de la nage ou dans le calcul statistique des effets positifs des nouvelles inoculations, avant les travaux de Jenner sur la vaccine. La tentation de présenter un Franklin-précurseur est évitée par une contextualisation minutieuse des débats, dans laquelle apparaît un Franklin boulimique de progrès, se plaçant au centre des cercles d'influence politiques et scientifiques les plus larges.

${ }^{1}$ Rédacteur en chef du Journal for the History of the Neurosciences.

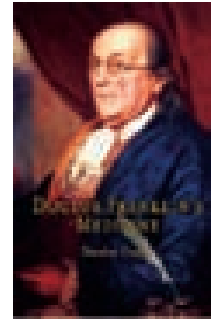

CNRS UMR 7102, UPMC Case 14,

7, quai Saint Bernard,

75005, Paris. Club d'Histoire

des Neurosciences de la Société

des Neurosciences,

http://jeangael.barbara.free.fr

jean-gael.barbara@snv.jussieu.f

La création par Franklin d'un hôpital pour les aliénés est rapportée dans un quatrième chapitre et apparaît dans l'ordre de l'Europe du XVIII siècle. Les relations privilégiées avec l'Écosse, concernant les matières médicales, apparaissent dans l'adoption pour son hôpital du modèle de l'infirmerie d'édimbourg. Les essais thérapeutiques par l'électricité, réalisés à Philadelphie sur des patients atteints de paralysie ou d'hystérie, confirment le désir de Franklin d'expérimenter de nouvelles thérapeutiques avec l'espoir réel de guérir. Les mourants sont exclus, les aliénés perdus enfermés. Franklin semble être le premier à avoir expérimenté des chocs électriques sur le crâne, démontrant perte de conscience ou amnésie. Le détail des échecs et succès concernant ses plus célèbres patients révèle une démarche médicale pragmatique et lucide.

C'est le rôle politique de Franklin qui est développé dans la $2^{\mathrm{e}}$ partie de l'ouvrage (chapitres 7 à 11 ). Ses deux grandes missions diplomatiques en Angleterre (1757-1762; 1764-1775) furent en réalité des échecs. Alors âgé de plus de cinquante ans, élu l'année précédente à la Royal Academy de Londres, Franklin multiplie ses contacts et cherche à légitimer sa réputation. Le Franklin diplomate n'est pas seulement un représentant du Congrès, mais sert également d'ambassadeur à l'enseignement médical américain, en favorisant les échanges avec l'Angleterre et en recommandant des étudiants à l'Université Saint Andrew d'Édimbourg. C'est dans ce contexte que Finger analyse les opinions de Franklin sur la fièvre et la quinine, la qualité de l'air, la ventilation des hôpitaux ou encore l'intoxication par le plomb, tous sujets qui sont l'occasion d'un combat à mener. Le séjour suivant à Paris (1776-1785) montre que Franklin n'est pas prêt à adhérer sans discernement à toute théorie. Son refus de suivre les thèses du médecin Mesmer, pourtant soutenu par Marie-Antoinette, et au sujet duquel il préside une commission d'enquête voulue par Louis XVI, est un plaidoyer raisonné contre cette forme de charlatanisme.

Ce séjour en France donne lieu à l'analyse d'un Franklin s'immergeant rapidement dans le milieu scientifique parisien, dont il devient une figure incontournable, assistant aux réunions de l'Académie des Sciences et à celles d'une célèbre loge maçonnique, fréquentant Lavoisier, Voltaire, Cabanis ou Vicq-d'Azyr. Le savant habite en bordure de forêt un hôtel de Passy, et préfère les 
fourrures à la mode aristocratique. Voltaire le salue publiquement à l'Académie par une bise à la française, Turgot fait son éloge en latin. Ses combats sont la malnutrition de l'enfant, la thérapie par la musique ou l'adoption des lunettes à double foyer. Franklin plaît, mais la carrière scientifique heureuse de l'illustre savant est derrière lui. Cependant, Franklin est encore écouté au sujet de la goutte et des calculs de la vessie, deux questions qui l'occupent à la fois comme médecin et comme sujet souffrant de ces maux courants. Buffon lui-même lui demande conseil. Au Congrès, John Jay plaide finalement en faveur d'une retraite bien méritée et obtient en 1785 le retour en gloire d'un Franklin fatigué et malade.

Si Franklin ne fut peut-être pas un grand médecin, il fut une grande figure des cercles médicaux aux États-Unis, en Angleterre, en $́$ cosse et en France, par ses efforts, son influence politique et scientifique et ses ressources financières. Franklin fut un véritable diplomate de l'art de soigner, et surtout de prévenir les maladies, un intermédiaire irremplaçable et souvent ingénieux entre les pays, les institutions et les hommes de différentes conditions. $\diamond$

\section{RÉFÉRENCE}

1. Finger S. Doctor Franklin's Medicine. Philadelphie : University of Pennsylvania Press, $2006: 384$ p.

\section{TIRÉS À PART}

J.G. Barbara

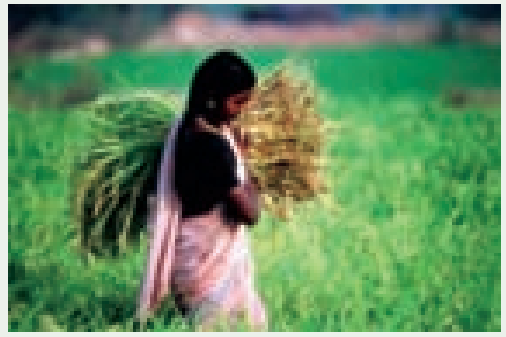

\section{FLASH}

\section{Un riz résistant aux inondations Nouveau progrès pour la lutte contre la faim dans le monde}

Le riz est un aliment vital pour une grande partie de l'humanité, non seulement en Asie, mais aussi en Amérique Latine et en Afrique. Mais pour satisfaire la demande de riz au cours des trente prochaines années, il est indispensable d'augmenter la production et de rechercher des variétés de riz - on connaît environ 120000 variétés d'Orysa sativa - dont le rendement soit supérieur aux variétés traditionnelles. Le riz semi-nain développé aux Philippines et les formes hybrides de la Révolution Verte en Chine (qui occupent actuellement environ la moitié de la superficie totale des rizières chinoises) répondent en partie à ce défi. En 40 ans, ces progrès ont permis un doublement de la production. Mais, en Asie, la population augmentant de 56 millions de personnes par an, il est essentiel de trouver encore d'autres moyens pour augmenter la production, qui devrait atteindre 770 millions en 2025 pour faire face aux besoins de ce seul continent.

Le séquençage du génome du riz s'est terminé en 2005. II laissait espérer de nouveaux progrès. C'est chose faite avec la découverte des gènes permettant une meilleure résistance à l'immersion prolongée. En effet, si le riz doit être cultivé dans des terres irriguées, il ne supporte pas l'anaérobiose par submersion prolongée. Or, il s'agit malheureusement d'un phénomène fréquent pendant les moussons dans les grands deltas d'Asie comme celui du Gange, et au Bangladesh, où chaque année des pluies torrentielles détruisent de nombreuses cultures, un désastre pour des populations parmi les plus pauvres de la planète.

On savait que certains cultivars de 0 . sativa indica supportaient d'être submergés jusqu'à 15 jours. Des travaux récents avaient montré que cette tolérance à l'immersion était liée à un locus, Submergencel (Subl) sur le chromosome 9 [1]. Une étude ultérieure y a mis en évidence trois domaines, SublA, SublB et SubIC [2]. Puis, au niveau de ce locus, trois gènes ont été isolés qui codent des facteurs de réponse à l'éthylène ( $\varepsilon R F)$ [3]. Deux de ces gènes sont invariables dans toutes les espèces de riz, seul SublA varie selon les espèces (et n'existe pas dans le génome du riz japonica). Chez les plantes, les domaines ERF sont connus pour contrôler la réponse à des stress biotiques ou abiotiques. Une induction rapide de l'expression de SublA au cours de la submersion en faisait un bon candidat en tant que déterminant génétique. Un criblage, mené sur 17 haplotypes indica et 4 haplotypes japonica a permis d'identifier deux allèles SublA, SublA-1 chez des espèces tolérantes et SublA-2 chez les espèces intolérantes. Ces deux allèles différent par un seul nucléotide, responsable de la substitution en position 556 d'une proline par une sérine chez les espèces tolérantes. La phosphorylation de cette sérine stimule ainsi un gène $A d h$ (alcool déshydrogénase) fonctionnel en milieu anaérobie. Le rôle déterminant de SublA-l est aussi prouvé par le fait que sa surexpression dans une souche intolérante de 0 . sativa sp. japonica induit une réaction de tolérance. Comme chez les riz tolérants, il y a alors dépression temporaire de l'expression de SubIC et stimulation de celle de Adh. En utilisant des marqueurs, le locus a été incorporé à des souches largement cultivées en Asie. Fait important, la résistance à la submersion aqueuse ne modifie pas le rendement de la culture. On peut en espérer une sécurisation des récoltes dans les régions périodiquement inondées. II est intéressant de noter que l'haplotype sublA-l a été retrouvé au Sri Lanka et dans les régions orientales de l'Inde, ce qui laisse supposer que, venant de plus de $1000 \mathrm{~km}$, il aurait été introduit dans les variétés locales et s'y serait maintenu, démontrant ainsi sa valeur en agronomie. Au vu de ces résultats, un scientifique japonais rapproche les éléments EFR d'éléments DFR, répondant à un stress de déshydratation, les uns et les autres codant pour le facteur de régulation des plantes AP2 [4]. La tolérance à la submersion induite par SublA pourrait donc aussi aider à comprendre la résistance à d'autres stress abiotiques. Ainsi, comme on l'escomptait, la connaissance du génome du riz (400 millions de bases d'ADN contenant 37544 gènes localisés sur 12 chromosomes) est source de fécondité.

1. Xu K, MacGill DJ. Mol Breed $1996 ; 2: 219-24$.

2. Xu K. et al. Mol Gen Genet $2000 ; 263: 681-9$.

3. Xu K. et al. Nature $2006 ; 442: 705-8$.

4. Sasaki T. Nature $2006 ; 442: 635-6$.

Dominique Labie 